\title{
Identification of Vinculin as a Potential Diagnostic Biomarker for Acute Aortic Dissection Using Label-Free Proteomics
}

\author{
He-Qing Wang $\mathbb{D}^{1},{ }^{1}$ Hang Yang, ${ }^{1}$ Qian Tang, ${ }^{1}$ Yi-Chen Gong, ${ }^{1}$ Yuan-Hao Fu, ${ }^{1}$ Feng Wan, \\ Bo Yang, ${ }^{1}$ Rui Guo, ${ }^{1}$ Yong-Liang Zhong, ${ }^{2}$ Jun-Ming Zhu, ${ }^{2}$ and Zhe Zhang ${ }^{1}$ \\ ${ }^{1}$ Department of Cardiac Surgery, Peking University Third Hospital, Beijing 100191, China \\ ${ }^{2}$ Department of Cardiovascular Surgery, Beijing Aortic Disease Center, Beijing Anzhen Hospital, Capital Medical University, \\ Beijing 100020, China
}

Correspondence should be addressed to Zhe Zhang; zhangzhe@bjmu.edu.cn

Received 16 March 2020; Revised 4 June 2020; Accepted 3 July 2020; Published 20 July 2020

Academic Editor: Kibret Mequanint

Copyright ( $2020 \mathrm{He}$-Qing Wang et al. This is an open access article distributed under the Creative Commons Attribution License, which permits unrestricted use, distribution, and reproduction in any medium, provided the original work is properly cited.

\begin{abstract}
Acute aortic dissection (AAD) is an emergent vascular disease. Currently, its diagnosis depends on clinical and radiological investigations but lacking of serum biomarkers. In this study, we aimed to identify potential serum biomarkers for AAD using label-free proteomics approach. A total of 90 serum samples were collected from three groups: patients with acute aortic dissection (AAD, $n=30$ ), patients with acute myocardial infarction (AMI, $n=30$ ), and healthy controls $(n=30)$, and the first four samples from each group were selected for label-free proteomics analysis. Using label-free approach, a total of 22 differentially expressed proteins were identified in the serum samples of the AAD group, of which 15 were upregulated and 7 were downregulated as compared to the AMI and healthy control groups. The most prominent increased protein was vinculin, which was selected to validate in total samples. The level of vinculin was significantly elevated in AAD patients $(15.8 \mathrm{ng} / \mathrm{ml}$, IQR: $9.3-19.9 \mathrm{ng} / \mathrm{ml})$ than that in AMI patients $(8.6 \mathrm{ng} / \mathrm{ml}$, IQR:5.3-11.4 $\mathrm{ng} / \mathrm{ml})$ and healthy volunteers (5.3 ng/ml, IQR:2.8$7.6 \mathrm{ng} / \mathrm{ml}), P<0.0001$. Furthermore, the concentration of vinculin both increased in type A and B dissection. At the early stage of $\mathrm{AAD}$, vinculin maintained a high level to 48 hours compared with that of AMI. Our study demonstrated that vinculin may play a role in the early diagnosis of AAD.
\end{abstract}

\section{Introduction}

Acute aortic dissection (AAD) is the most common thoracic aortic emergency and may be rapidly fatal without early diagnosis and appropriate management [1]. For untreated A type of $\mathrm{AAD}$, the mortality rate increased by $1-2 \%$ every 1 hour [2]. Therefore, it is essential to improve the survival rate of AAD by early rapid diagnosis. The main challenge of diagnosis for $\mathrm{AAD}$ is to distinguish it from sudden chest pain caused by other diseases (especially acute myocardial infarction, pulmonary embolism, etc.), because the treatment between these patients is completely different or even the opposite, such as when AAD is misdiagnosed as AMI, incorrect use of antiplatelet therapy can increase the risk of further tearing or even rupture of the aorta $[3,4]$.
Highly sensitive and specific circulating biomarkers are essential for accelerating the diagnosis of AAD. In recent years, markers have been focused on vascular smooth muscle cell injury, including smooth muscle myosin heavy chain, calponin, and creatine kinase isoenzyme BB; extracellular matrix damage, including matrix metalloproteinases, and soluble elastin degradation fragments; and D-dimer released by activation of coagulation and fibrinolysis system [5]. However, none of these biomarkers are widely available with high sensitivity and specificity. Currently, proteomics has become a reliable method for screening early diagnostic markers [6-8]. Compared with traditional absolute isotope labeling (iTRAQ), label-free proteomics can identify more low-abundance proteins which are generally valuable diagnostic biomarkers $[9,10]$. With this method, we aim to 
screen out potential circulating biomarkers which may be released due to the damage of aortic media.

\section{Materials and Methods}

2.1. Patients Enrollment. A total of $30 \mathrm{AAD}$ patients, $30 \mathrm{AMI}$ patients, and 30 healthy volunteers were consecutively enrolled from 2018 to 2019 in our hospital. Label-free analysis was performed in the first eight patients (4 AAD and 4 AMI) and four healthy individuals. The time interval of from onset of chest/back/abdominal pain to admission in hospital was less than 48 hours. The diagnosis of AAD was followed by 2014 European guidelines and confirmed by computed tomographic arteriography (CTA) indicating double-lumen sign. AMI was confirmed by elevated electrocardiography (ECG) and cardiac troponin T (cTNT). The protocol was approved by the Ethics Committee of our hospital and all subjects signed informed consent for the study.

$10 \mathrm{ml}$ blood samples were collected immediately after admission and centrifuged at $3000 \mathrm{rpm}$ for $10 \mathrm{~min}$ at $4^{\circ} \mathrm{C}$. The serum was then frozen at $-80^{\circ} \mathrm{C}$ until further tests.

\subsection{Experimental Methods}

2.2.1. Proteomics Technology. 14 high abundance proteins in the serum were removed using Human 14 Multiple Affinity Removal System (MARS, agilent, USA). $20 \mu \mathrm{g}$ of disposed sample was perfused in $4-12 \%$ gradient gel (4-12\% BisTrisNuPAGE gel) and MOPS protein separation buffersystem. The gel was divided into 40 equal fractions and subjected to enzymatic hydrolysis, rinsed with $25 \mathrm{mmol} / \mathrm{L}$ sodium hydrogencarbonate, and then dehydrated and dried by acetonitrile. The sample was added with $10 \mathrm{mmol} / \mathrm{L}$ dithiothreitol in a water bath at $60^{\circ} \mathrm{C}$, alkylated with $50 \mathrm{mmol} / \mathrm{L}$ iodoacetamide for $1 \mathrm{~h}$ at room temperature, digested by trypsin at $37^{\circ} \mathrm{C}$ for $4 \mathrm{~h}$, added with $50 \%$ acetonitrile $/ 2 \%$ formic acid for vacuum centrifuge, and the supernatant was taken for LC-MS/MS analysis. Mass spectrometry was performed by the NanoAcquity HPLC-LTQ-Orbitrap Velos system (AB SCIEX, Canada). The chromatographic column $(\mathrm{C} 18,50 \times 2.1 \mathrm{~mm}$, $1.7 \mu \mathrm{m}$ ) was eluted in a $75 \mu \mathrm{m}$ analytical column at a flow rate of $350 \mathrm{nl} / \mathrm{min}$. The mobile phase A was an aqueous solution containing $0.1 \%$ formic acid, and the mobile phase B was eluted with a gradient of acetonitrile containing $0.1 \%$ formic acid. The gradient program was set to $0-3 \mathrm{~min} 90 \% \mathrm{~B}, 3-$ $15 \min 90 \%-40 \% \mathrm{~B}, 15-16 \min 40 \%-2 \% \mathrm{~B}, 16-18 \min 2 \% \mathrm{~B}$, $18-19 \min 2 \%-90 \% \mathrm{~B}, 19-23 \mathrm{~min} 90 \% \mathrm{~B}$. The ion source was DuoSpray with electrospray ionization mode for scanning analysis. The negative ion spray voltage was set to $-4500 \mathrm{~V}$, and the Turbo spray temperature was $450^{\circ} \mathrm{C}$; the positive ion spray voltage was set to $4500 \mathrm{~V}$, and the Turbo spray temperature was $450^{\circ} \mathrm{C}$. The precursor ions were scanned by electrostatic orbitrap MS, 15 of which with the highest peaks were selected for MS/MS scan.

2.2.2. Validation of Candidate Biomarkers. D-dimer was tested on a Beckman ACL TOP 700 Automated Coagulation Analyzer by a commercial latex-enhanced immunoturbidimetric assay (HemosIL D-dimer HS, Instrumentation Laboratory, USA). Vinculin was detected using a human ELISA kit (EKU08145, Biomatik, Canada). All procedures were operated in strict accordance with the standard instructions. Duplication of each sample was validated.

2.3. Statistical Analysis. The MS raw data were identified in the Swissport Human database by the search software Mascot software (Matrix Science Ltd, USA), and the results were verified by the Scaffold algorithm. 1.5-fold change (FC) of LFQ-intensity between the AAD and control groups was set as the cut off value in differential protein screening, indicating that $\mathrm{FC} \geq 1.5$ is upregulated and $\mathrm{FC} \leq 0.667$ is downregulated. The categorical variables were indicated as percentage, and the continuous variables were indicated as mean \pm standard deviation or median (interquartile range). Comparative analysis of multiple groups was performed with independent sample $t$-test, one-way analysis of variance, rank sum test, or chisquare test. Statistical significance was defined as $P<0.05$.

\section{Results}

3.1. Clinical Characteristics. $30 \mathrm{AAD}$ patients (22 with type A, 8 with type B, 5 with Marfan syndrome, 4 with aortic bicuspid valve malformation), 30 AMI patients, and 30 healthy controls were enrolled (see Table 1). 4 subjects of each group were selected for proteomic analysis. The age of AAD patients was significantly lower than that of the AMI group ( $48.8 \pm 9.9$ vs. $63.7 \pm 10.8, P<0.0001)$. Blood sample was collected from patients with AAD and AMI at admission immediately $(17.3 \pm 10.6$ hours vs. $13.5 \pm 9.6$ hours, $P=$ $0.157)$. There was no significant difference in gender composition among the three groups and in the prevalence of hypertension between AAD and AMI groups.

3.2. Proteomics Results and Bioinformatics Analysis. A total of 1096 serum proteins were identified by searching the Swissprot Human database with the Mascot software, and 22 differentially expressed proteins were screened in the serum of the AAD group, 15 of which were upregulated ( $>1.5$ fold) and 7 were downregulated $(<0.67$ fold) as compared to the AMI and healthy control groups. Based on their biofunction classification, the major biological process types included cell adhesion (27\%), extracellular matrix organization (19\%), angiogenesis (17\%), signal transduction (15\%), inflammation response (13\%), and cell proliferation (9\%) proteins (see Figure 1(a)). A network was constituted by protein-protein interaction analysis on the string website (see Figure 1(c)). In addition, the differentially expressed proteins were involved in P13K-AKT signaling (21\%), focal adhesion (20\%), proteoglycans in cancer (18\%), PPAR signaling (17\%), regulation of actin cytoskeleton (15\%) and complement, and coagulation cascades (9\%) pathways (see Figure 1(b)). Vinculin, a kind of cytoskeletal protein, increased most significantly among the differential proteins $(\mathrm{AAD} / \mathrm{AMI}$ ratio $=4.85$, $\mathrm{AAD} / \mathrm{Ctl}$ ratio $=8.33)$ (see Table 2).

3.3. Validation of Candidate Biomarkers. Based on the proteomics and bioinformatics results, vinculin was selected as a candidate biomarker and validated in total samples; moreover, D-dimer was selected as the gold standard which is recommended in the guideline. The concentration of 
TABLe 1: Clinical characteristics of total subjects.

\begin{tabular}{|c|c|c|c|c|}
\hline & $\mathrm{AAD}$ & AMI & Healthy Ctrls & $P$ value \\
\hline$n$ & 30 & 30 & 30 & \\
\hline Age $($ mean \pm SD) & $48.8 \pm 9.9$ & $63.7 \pm 10.8$ & $47.3 \pm 9.5$ & $0.0001^{\mathrm{a}}$ \\
\hline Gender, male (\%) & $21(70.0 \%)$ & $20(66.7 \%)$ & $21(70.0 \%)$ & $0.95^{\mathrm{b}}$ \\
\hline Hours from onset to admission (mean \pm SD) & $17.3 \pm 10.6$ & $13.5 \pm 9.6$ & l & $0.157^{\mathrm{c}}$ \\
\hline Hypertension (\%) & $21(70.0 \%)$ & $19(63.3 \%)$ & 1 & $0.785^{\mathrm{b}}$ \\
\hline Smoking (\%) & $9(30.0 \%)$ & $10(33.3)$ & l & $1.000^{\mathrm{b}}$ \\
\hline Type A (\%) & $22(73.3 \%)$ & I & I & l \\
\hline Marfan syndrome (\%) & $5(15.0)$ & l & l & l \\
\hline Bicuspid valve (\%) & $4(13.3)$ & l & l & l \\
\hline D-dimer (ng/mL) (median, IQR) & $2886.3(1737.2-4362.1)$ & $1552.0(465.1-2327.0)$ & $269.5(174.3-394.3)$ & $0.0001^{\mathrm{d}}$ \\
\hline
\end{tabular}

${ }^{\mathrm{a}}$ : one-way analysis of variance; ${ }^{\mathrm{b}}$ : chi-square test; ${ }^{\mathrm{c}}$ : t-test, ${ }^{\mathrm{d}}$ : Kruskal-Wallis test, D-dimer was indicated as median (IQR), and the rest of continuous variable were indicated as mean \pm SD. AAD: acute aortic dissection; AMI: acute myocardial infarction.

vinculin $(15.8 \mathrm{ng} / \mathrm{ml}, \mathrm{IQR}: 9.3-19.9 \mathrm{ng} / \mathrm{ml})$ was significantly higher in AAD patients than AMI group $(8.6 \mathrm{ng} / \mathrm{ml}$, IQR: $5.3-11.4 \mathrm{ng} / \mathrm{ml}$ ) and healthy control group $(5.3 \mathrm{ng} / \mathrm{ml}$, IQR: $2.8-7.6 \mathrm{ng} / \mathrm{ml}), P<0.0001$. Although the concentration of vinculin showed a higher trend in the AMI group compared with the healthy group, the difference was not statistically significant. Meanwhile, the concentration of D-dimer was significantly higher in AAD patients $(2886.3 \mathrm{ng} / \mathrm{ml}$, IQR: 1737.2-4362.1) than AMI group (1552.0 ng/ml, IQR465.12327.0) and healthy control group $(269.5 \mathrm{ng} / \mathrm{ml}$, IQR: 174.3-394.3), $P<0.0001$ (see Table 3 and Figure 2).

3.4. Diagnostic Performance of Candidate Biomarkers. There are reports that some markers may express different concentrations between type A and $\mathrm{B}$ dissection due to involvement of different aortic sections [11]; therefore, we further analyzed the concentration of vinculin in different dissection types which demonstrated that the concentration of vinculin was $15.8 \mathrm{ng} / \mathrm{ml}$ (IQR: 9.1-20.7) and $14.7 \mathrm{ng} / \mathrm{ml}$ (IQR: 9.2-17.5) in type $\mathrm{A}$ and $\mathrm{B}$ dissection, respectively, both higher than control groups, and no significant difference was discovered between the two dissections (see Figure 3(a)).

Time course was also analyzed from onset to blood collection (time windows of 0 to 12,12 to 24 , and 24 to 48 hours) which showed that the concentration of vinculin increased at an early stage of AAD and maintained a high level to 48 hours compared with that of AMI (see Figure 3(b)).

\section{Discussion}

As a lethal cardiovascular emergency, early rapid diagnosis is essential to improve AAD patient survival [12]. Imaging facilities are pivotal for the diagnosis and surgical treatment of AAD [13]. However, high sensitive and specific AAD biomarkers can help to make the choice of CTA, just similar to troponin of coronary angiography for AMI [14], which can increase the diagnostic pretest probability for the patients with acute chest pain at emergency department. Therefore, biomarkers can shorten the time from admission to surgery. Moreover, for some community hospitals or remote areas which have no facilities, biomarkers can help to decide whether to transfer patients to superior hospital or not.

Proteomics technology has recently become a reliable method for screening disease diagnostic markers $[15,16]$. It has been reported that an extracellular matrix proteinlumican was identified by comparing the serum proteomics between the AAD and control groups using iTRAQ technology, but this marker lacked further analysis of its level in different dissection types and time windows [8]. Label-free proteomics, unlike traditional iTRAQ technology, does not depend on isotope labeling and mix of all samples which can accurately reflect individual differences and identifying more low-abundance proteins in serum which contains multiple orders of magnitude abundance proteins $[10,17]$. In this study, we first used label-free proteomics to screen for early diagnosis markers of AAD. Based on the previous reports [18], 1.5-fold change (FC) of LFQ-intensity between the $\mathrm{AAD}$ and control groups was set as the cut off value in differential protein screening, and 22 differential expressed proteins were identified. Notably, vinculin, a kind of cytoskeletal proteins, increased most significantly among the differential proteins $(\mathrm{AAD} / \mathrm{AMI}$ ratio $=4.85, \mathrm{AAD} / \mathrm{Ctl}$ ratio $=8.33$ ). Further validation of total samples showed that the concentration of vinculin in AAD was 1.8 and 3 times higher than that of the AMI and healthy control groups, respectively.

Different types (A and B) of AAD can present various clinical symptoms, and it is reported that the concentrations of several diagnostic markers were lower in type B dissection than that in the A type (such as TGF- $\beta$ ) which limited its availability [19]. In our study, the concentration of vinculin in type A and B dissections showed no significant difference, both higher than that of all controls, indicating that vinculin has a favorable diagnostic performance for both type A and B dissections. In addition, the time course of a biomarker is also a crucial factor, which increases too slowly or decreases too fast will limit its application. Therefore, we analyzed the change of concentration of vinculin from onset which showed it increased rapidly in the early stage $(<12$ hours) and then maintained at a higher level for 48 hours in AAD 


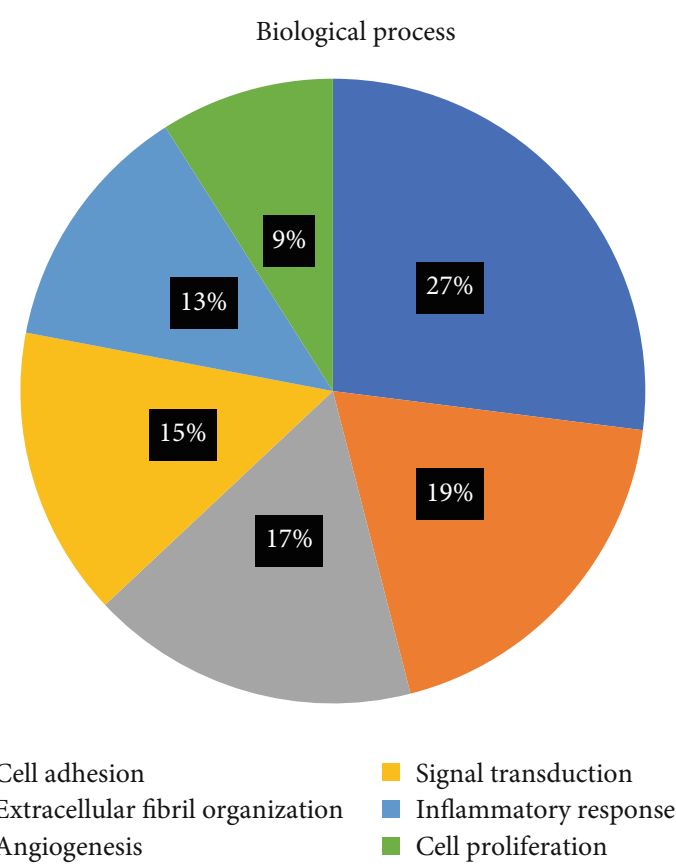

(a)

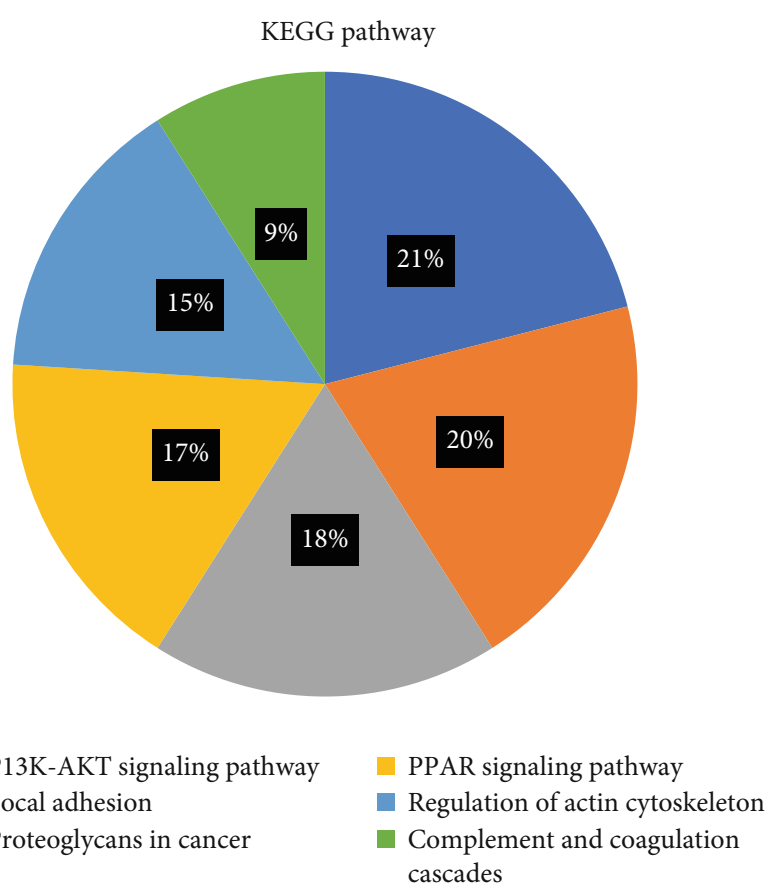

(b)

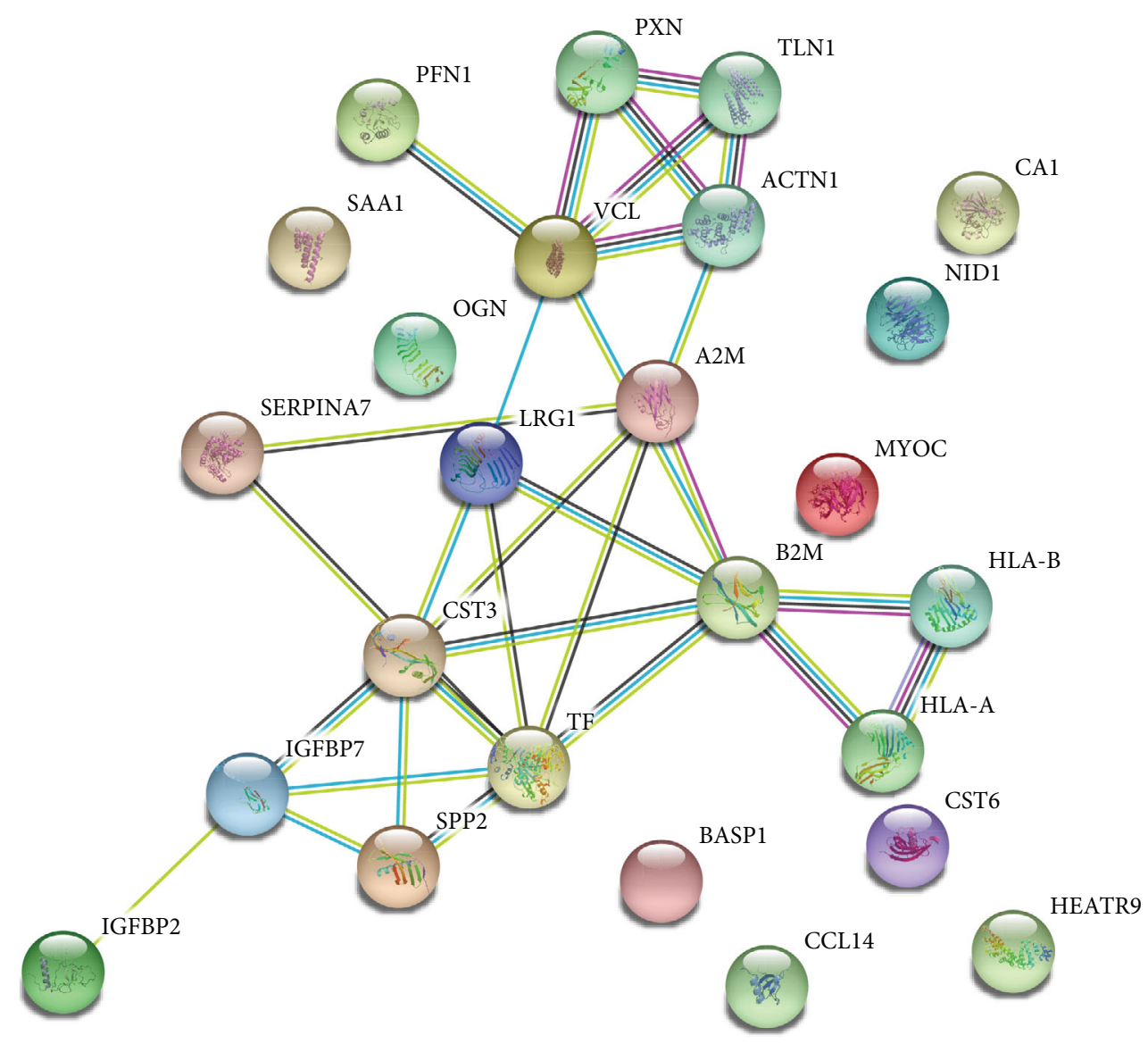

(c)

Figure 1: Bioinformatics analysis of the differential proteins. (a) Biological process. (b) Pathway. (c) Network of proteins. 
TABLE 2: Differentially expressed proteins $(\mathrm{FC}>1.5$ or $<0.667)$ in AAD identified by label-free proteomics.

\begin{tabular}{|c|c|c|c|c|c|}
\hline Accession & $\begin{array}{l}\text { Gene } \\
\text { name }\end{array}$ & Protein name & Biological process & $\begin{array}{l}\mathrm{AAD} / \mathrm{AMI} \\
\text { ratio }\end{array}$ & $\begin{array}{l}\mathrm{AAD} / \mathrm{HC} \\
\text { ratio }\end{array}$ \\
\hline \multicolumn{6}{|l|}{$\begin{array}{l}\text { Increased in } \\
\text { AAD }\end{array}$} \\
\hline P18206 & VCL & Vinculin & Adherens junction assembly & 4.85 & 8.33 \\
\hline P0DJI8 & SAA1 & Serum amyloid A-1 protein & Activation of MAPK activity & 2.36 & 5.58 \\
\hline Q16627 & CCL14 & $\mathrm{C}-\mathrm{C}$ motif chemokine 14 & Cellular calcium ion homeostasis & 4.61 & 4.89 \\
\hline P01034 & CST3 & Cystatin-C & Extracellular fibril organization & 2.15 & 4.47 \\
\hline Q16270 & IGFBP7 & $\begin{array}{l}\text { Insulin-like growth factor-binding } \\
\text { protein } 7\end{array}$ & Cell adhesion & 2.12 & 4.44 \\
\hline Q99972 & MYOC & Myocilin & $\begin{array}{l}\text { Positive regulation of focal adhesion } \\
\text { assembly }\end{array}$ & 3.86 & 4.14 \\
\hline P18065 & IGFBP2 & $\begin{array}{l}\text { Insulin-like growth factor-binding } \\
\text { protein } 2\end{array}$ & Aging & 4.62 & 3.98 \\
\hline P98160 & HSPG2 & LG3 peptide & Angiogenesis & 3.85 & 3.76 \\
\hline Q15828 & CST6 & Cystatin-M & Anatomical structure morphogenesis & 2.22 & 2.96 \\
\hline P20774 & OGN & Mimecan & $\begin{array}{l}\text { Negative regulation of smooth muscle cell } \\
\text { proliferation }\end{array}$ & 3.45 & 2.79 \\
\hline P07737 & PFN1 & Profilin-1 & Actin cytoskeleton organization & 2.95 & 2.48 \\
\hline P80723 & BASP1 & Brain acid soluble protein 1 & Diaphragm development & 1.57 & 2.45 \\
\hline P02750 & LRG1 & Leucine-rich alpha-2-glycoprotein & Brown fat cell differentiation & 1.74 & 2.36 \\
\hline P61769 & $\mathrm{B} 2 \mathrm{M}$ & Beta-2-microglobulin & Antibacterial humoral response & 2.24 & 2.06 \\
\hline P14543 & NID1 & Nidogen-1 & Basement membrane organization & 1.53 & 1.73 \\
\hline \multicolumn{6}{|c|}{$\begin{array}{l}\text { Decreased in } \\
\text { AAD }\end{array}$} \\
\hline Q13103 & SPP2 & Secreted phosphoprotein 24 & Bone remodeling & 0.51 & 0.24 \\
\hline P00915 & CA1 & Carbonic anhydrase 1 & Bicarbonate transport & 0.27 & 0.29 \\
\hline P05543 & SERPINA7 & Thyroxine-binding globulin & Thyroid hormone transport & 0.24 & 0.33 \\
\hline P01871 & IGHM & Ig mu chain $\mathrm{C}$ region & Adaptive immune response & 0.58 & 0.42 \\
\hline A2RTY3 & HEATR9 & Protein HEATR9 & $\begin{array}{l}\text { Hematopoietic progenitor cell } \\
\text { differentiation }\end{array}$ & 0.54 & 0.45 \\
\hline P02787 & $\mathrm{TF}$ & Serotransferrin & Cellular iron ion homeostasis & 0.42 & 0.47 \\
\hline P01023 & $\mathrm{A} 2 \mathrm{M}$ & Alpha-2-macroglobulin & Blood coagulation, intrinsic pathway & 0.46 & 0.56 \\
\hline
\end{tabular}

AAD: acute aortic dissection; AMI: acute myocardial infarction.

TABLE 3: The serum concentrations of vinculin and D-dimer in each group.

\begin{tabular}{lcccc}
\hline & AAD $(n=30)$ & AMI $(n=30)$ & HC $(n=30)$ & $P$ value \\
\hline Vinculin $(\mathrm{ng} / \mathrm{mL})^{\mathrm{a}}$ & $15.8(9.3-19.9)$ & $8.6(5.3-11.4)$ & $5.29(2.8-7.6)$ & $<0.0001^{\mathrm{b}}$ \\
D-dimer $(\mathrm{ng} / \mathrm{mL})^{\mathrm{a}}$ & $2886.3(1737.2-4362.1)$ & $1552.0(465.1-2327.0)$ & $269.5(174.3-394.3)$ & $<0.0001^{\mathrm{b}}$ \\
\hline
\end{tabular}

${ }^{a}$ : median (interquartile range); ${ }^{\text {b }}$ Kruskal-Wallis test. AAD: acute aortic dissection; AMI: acute myocardial infarction; HC: healthy controls.

patients compared with AMI patients, indicating a satisfactory time window.

It is reported that the aortic wall is fragile in $\mathrm{AAD}$ due to media degeneration and elastin degradation, which cannot bear overloaded pressure stimulation, leading to tear of aortic wall [20]. Vinculin is a key protein locating at focal adhesion that regulates mechanochemical pathway of extracellular matrix and cytoskeleton $[21,22]$, which was found overexpressed in AAD according to our study, may contribute to abnormal migration, adhesion, and proliferation of vascular smooth muscle cells, switching them from contractile phenotype to synthetic phenotype. Synthetic vascular smooth muscle cells can secrete increasing MMPs, leading to imbalance of MMPs and TIMPs, ultimately resulting in proteolysis of elastin in aortic wall [23].

The maintenance of vessel homeostasis relies on the normal contractile function of vascular smooth muscle cells, which is closely related to cell-matrix adhesion. The extracellular mechanical signal (blood stimulation) is converted into intracellular chemical signal via matrix-membrane integrin- 


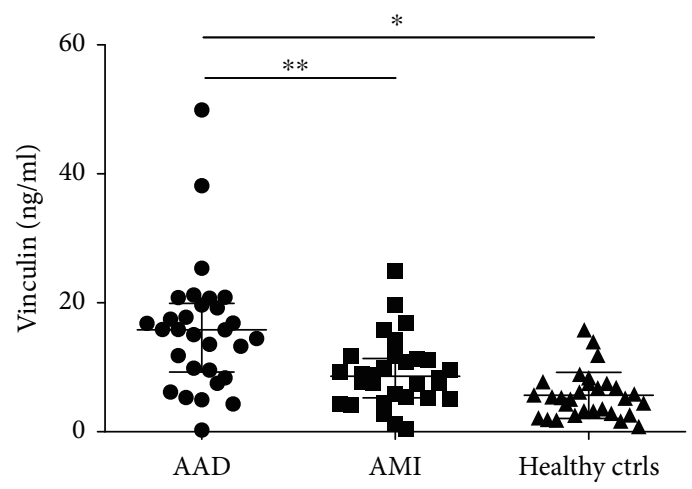

(a)

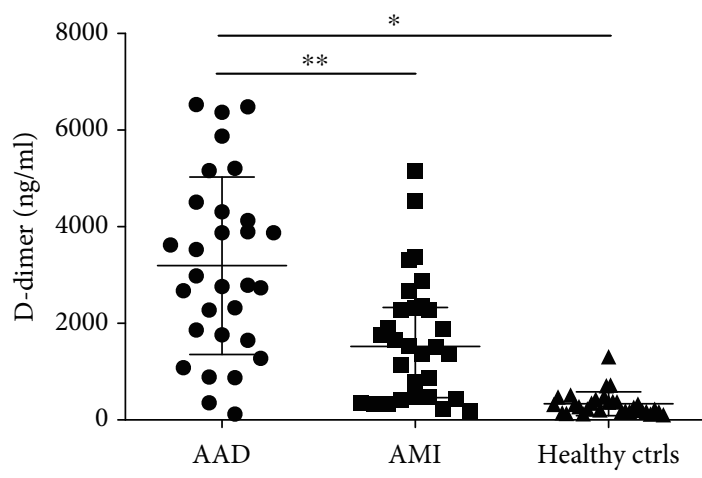

(b)

Figure 2: Validation of candidate biomarkers in serum samples. (a) Vinculin. (b) D-dimer. Levels of these candidate biomarkers were measured in serum of $\operatorname{AAD}(n=30)$, AMI $(n=30)$, and healthy controls $(n=30)$. Median values are shown by a horizontal line, and the error bars represent the interquartile range of measurements for 30 samples in three groups. $P$ values were calculated with Kruskal-Wallis test. $* P<0.0001, * * P<0.0001$.

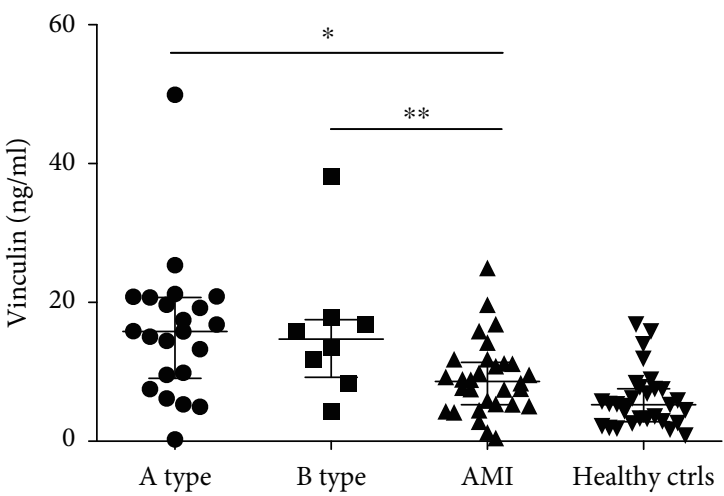

(a)

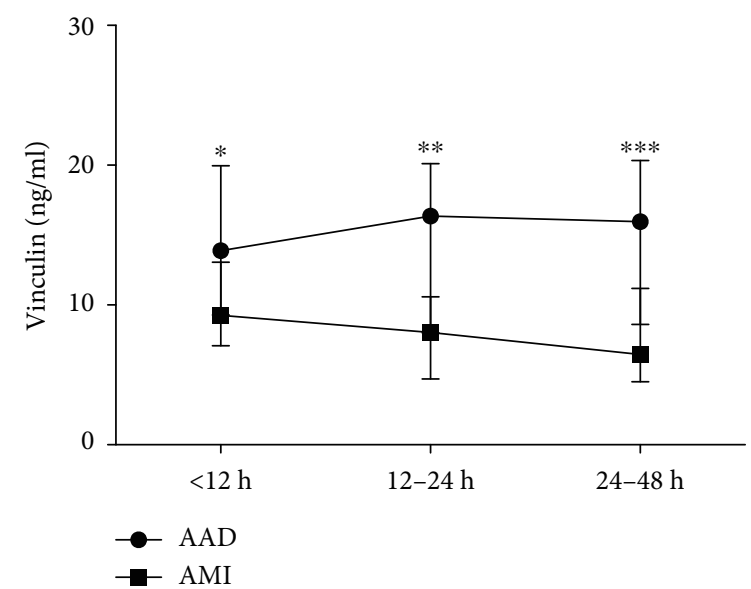

(b)

Figure 3: Subanalysis the concentration of vinculin according to type of dissection and time course. (a) Type of dissection (type A = 22, type $\mathrm{B}=8$ ). (b) Time windows from onset ( 0 to 12,12 to 24 , and 24 to 48 hours). Median values are shown by a horizontal line and the error bars represent the interquartile range of measurements in each group. $P$ values were calculated with Kruskal-Wallis test. $*, * *, * * *$ $P<0.05$.

focal adhesion pathway, which can further activate the polymerization of downstream actin, stabilize the cytoskeleton, and maintain the plasticity of blood vessel [24]. Therefore, mutations in genes encoding cell-matrix adhesion-related proteins (FBN-1, MFAP5, and COL3A1) can lead to AAD [25]. Our further GO enrichment and KEGG pathway analysis of proteomics results indicated that the most dominant biological process of differential proteins was cell adhesion $(27 \%)$ in our study, which was consistent with previous proteomic analysis suggesting dysfunction of cell adhesion and matrix remodeling in AAD patients revealing a potential pathophysiological mechanism [18].

D-dimer is the only diagnostic marker in 2014 European guideline (IIa recommendation) [26], which was selected as a comparative marker in our study. The concentration of Ddimer in AAD was nearly 1.8 and 10 times higher than that of the AMI and healthy control groups, respectively, which was consistent with previous reports [27, 28]. D-dimer is the degradation product of thrombus, and its elevation suggests that the activation of blood coagulation and fibrinolysis system generally occurring in DIC, pulmonary embolism, cerebral infarction, myocardial infarction, etc. [28]. In AAD, blood flow enters into the media of aorta through the tear of the intima, activating the coagulation system, thrombosing in false lumen, subsequently fibrin degrading and releasing D-dimer into the blood circulation [29]. Recently, it is reported that $\mathrm{D}$-dimer can play a potential role in activating MMPs [30]. Thus, vinculin and D-dimer may both anticipate the pathophysiology of tissue degradation during $A A D$ via the pathway of MMPs, which is exactly the further investigation purpose in our study.

To our knowledge, the study provides the first evidence that vinculin is associated with AAD and could be a novel biomarker for the early diagnosis. However, there are two 
limitations in our study. First of all, we did not validate all proteins which were identified in proteomics analysis, especially involved in cell adhesion and focal adhesion. On the other hand, our control groups only enrolled AMI patients but lacking other diseases presenting chest/back pain, especially pulmonary embolism which is easily confused with AAD in differential diagnosis. Therefore, a large prospective cohort should be further validated in suspected patients with $\mathrm{AAD}$ to evaluate the clinical application of vinculin.

\section{Conclusions}

A novel biomarker-vinculin was screened in AAD patients compared with AMI patients and healthy controls through label-free proteomics technology. Further validation of all subjects revealed vinculin was elevated significantly. Therefore, the present study will provide meaningful data and new ideas for the differential diagnosis of AAD.

\section{Data Availability}

The data used to support the findings of this study are available from the corresponding author upon request.

\section{Conflicts of Interest}

There are no conflicts of interest.

\section{Authors' Contributions}

He-Qing Wang and Hang Yang contributed equally to this work.

\section{Acknowledgments}

This study was supported by the "UMHS-PUHSC" Joint Institute for Translational and Clinical Research (2014JI053).

\section{References}

[1] D. P. Howard, A. Banerjee, J. F. Fairhead et al., "Populationbased study of incidence and outcome of acute aortic dissection and premorbid risk factor control: 10-year results from the Oxford Vascular Study," Circulation, vol. 127, no. 20, pp. 2031-2037, 2013.

[2] B. Iung, "New ESC guidelines: aortic disease," Heart, vol. 101, no. 6, pp. 421-423, 2015.

[3] J. W. Olin and V. Fuster, "Acute aortic dissection: the need for rapid, accurate, and readily available diagnostic strategies," Arteriosclerosis, Thrombosis, and Vascular Biology, vol. 23, no. 10, pp. 1721-1723, 2003.

[4] G. S. Van Arsdell, T. E. David, and J. Butany, "Autopsies in acute type A aortic dissection. Surgical implications," Circulation, vol. 98, 19 Supplement, pp. II299-II302, 1998.

[5] A. Segreto, A. Chiusaroli, S. De Salvatore, and F. Bizzarri, "Biomarkers for the diagnosis of aortic dissection," Journal of cardiac surgery, vol. 29, no. 4, pp. 507-511, 2014.

[6] D. Xu, Y. Li, X. Li et al., "Serum protein S100A9, SOD3, and MMP9 as new diagnostic biomarkers for pulmonary tuberculosis by iTRAQ-coupled two-dimensional LC-MS/MS," Proteomics, vol. 15, no. 1, pp. 58-67, 2015.
[7] W. Liu, Q. Yang, B. Liu, and Z. Zhu, "Serum proteomics for gastric cancer,” Clinica Chimica Acta, vol. 431, pp. 179-184, 2014.

[8] Z. Xiao, Y. Xue, C. Yao et al., "Acute aortic dissection biomarkers identified using isobaric tags for relative and absolute quantitation," BioMed Research International, vol. 2016, Article ID 6421451, 7 pages, 2016.

[9] L. Chang, J. Ni, J. Beretov et al., "Identification of protein biomarkers and signaling pathways associated with prostate cancer radioresistance using label-free LC-MS/MS proteomic approach," Scientific Reports, vol. 7, no. 1, p. 41834, 2017.

[10] P. Wu, F.-C. He, and Y. Jiang, "Label-free methods in quantitative proteomics," Acta Agronomica Sinica, vol. 40, no. 3, pp. 281-292, 2013.

[11] T. Suzuki, S. Trimarchi, D. Sawaki et al., "Circulating transforming growth factor-beta levels in acute aortic dissection," Journal of the American College of Cardiology, vol. 58, no. 7, p. 775, 2011.

[12] T. T. Tsai, S. Trimarchi, and C. A. Nienaber, "Acute aortic dissection: perspectives from the International Registry of Acute Aortic Dissection (IRAD)," European journal of vascular and endovascular surgery, vol. 37, no. 2, pp. 149-159, 2009.

[13] C. A. Nienaber and R. E. Clough, "Management of acute aortic dissection," The Lancet, vol. 385, no. 9970, pp. 800-811, 2015.

[14] S. Parve, B. A. Ziganshin, and J. A. Elefteriades, "Overview of the current knowledge on etiology, natural history and treatment of aortic dissection," The Journal of cardiovascular surgery, vol. 58, no. 2, pp. 238-251, 2017.

[15] A. Reumer, E. Maes, I. Mertens et al., "Colorectal cancer biomarker discovery and validation using LC-MS/MS-based proteomics in blood: truth or dare?," Expert Review of Proteomics, vol. 11, no. 4, pp. 449-463, 2014.

[16] Y. Cheng, C. Liu, N. Zhang, S. Wang, and Z. Zhang, "Proteomics analysis for finding serum markers of ovarian cancer," BioMed Research International, vol. 2014, Article ID 179040, 9 pages, 2014.

[17] K. A. Neilson, N. A. Ali, S. Muralidharan et al., "Less label, more free: approaches in label-free quantitative mass spectrometry," Proteomics, vol. 11, no. 4, pp. 535-553, 2011.

[18] K. Zhang, X. Pan, J. Zheng, D. Xu, J. Zhang, and L. Sun, “Comparative tissue proteomics analysis of thoracic aortic dissection with hypertension using the iTRAQ technique," European journal of cardio-thoracic surgery, vol. 47, no. 3, pp. 431-438, 2015.

[19] T. Suzuki, E. Bossone, D. Sawaki et al., "Biomarkers of aortic diseases," American heart journal, vol. 165, no. 1, pp. 15-25, 2013.

[20] D. M. Milewicz, K. M. Trybus, D. C. Guo et al., "Altered smooth muscle cell force generation as a driver of thoracic aortic aneurysms and dissections," Arteriosclerosis, thrombosis, and vascular biology, vol. 37, no. 1, pp. 26-34, 2017.

[21] T. Izard and D. T. Brown, "Mechanisms and functions of vinculin interactions with phospholipids at cell adhesion sites," The Journal of Biological Chemistry, vol. 291, no. 6, pp. 2548-2555, 2016.

[22] J. L. Bays and K. A. DeMali, "Vinculin in cell-cell and cellmatrix adhesions," Cellular and molecular life sciences: CMLS, vol. 74, no. 16, pp. 2999-3009, 2017.

[23] T. Kurihara, R. Shimizu-Hirota, M. Shimoda et al., "Neutrophil-derived matrix metalloproteinase 9 triggers acute aortic dissection," Circulation, vol. 126, no. 25, pp. 3070-3080, 2012. 
[24] Y. Hu, L. Lu, Z. Qiu, Q. Huang, Y. Chen, and L. Chen, "Mechanical stretch aggravates aortic dissection by regulating MAPK pathway and the expression of MMP-9 and inflammation factors," Biomedicine \& Pharmacotherapy, vol. 108, pp. 1294-1302, 2018.

[25] E. Gillis, L. Van Laer, and B. L. Loeys, "Genetics of thoracic aortic Aneurysm," Circulation research, vol. 113, no. 3, pp. 327-340, 2013.

[26] R. Erbel, V. Aboyans, C. Boileau et al., "2014 ESC Guidelines on the diagnosis and treatment of aortic diseases: document covering acute and chronic aortic diseases of the thoracic and abdominal aorta of the adult. The Task Force for the Diagnosis and Treatment of Aortic Diseases of the European Society of Cardiology (ESC)," European Heart Journal, vol. 35, no. 41, pp. 2873-2926, 2014.

[27] T. Suzuki, A. Distante, A. Zizza et al., "Diagnosis of acute aortic dissection by $\mathrm{D}$-dimer: the international registry of acute aortic dissection substudy on biomarkers (IRAD-Bio) experience," Circulation, vol. 119, no. 20, pp. 2702-2707, 2009.

[28] P. Nazerian, C. Mueller, A. M. Soeiro et al., "Diagnostic accuracy of the aortic dissection detection risk score plus D-dimer for acute aortic syndromes: the ADvISED Prospective Multicenter Study," Circulation, vol. 137, no. 3, pp. 250-258, 2018.

[29] J. S. Cui, Z. P. Jing, S. J. Zhuang et al., "D-dimer as a biomarker for acute aortic dissection: a systematic review and meta-analysis," Medicine, vol. 94, no. 4, article e471, 2015.

[30] K. Alexander, A. Banos, S. Abro et al., "Levels of matrix metalloproteinases in arthroplasty patients and their correlation with inflammatory and thrombotic activation processes," Clinical and Applied Thrombosis/Hemostasis, vol. 22, no. 5, pp. 441-446, 2015. 